\title{
Laparoscopic Reversal of Tubal Sterilization: A Demonstration Video
}

\author{
XIAOXI NIU ${ }^{1}$, Xiaolei Zhang ${ }^{2}$, Guiyu Zhang ${ }^{2}$, and Ming Yuan ${ }^{2}$ \\ ${ }^{1}$ Shandong University Qilu Hospital \\ ${ }^{2}$ Affiliation not available
}

May 5, 2020

\begin{abstract}
To describe the method in which fallopian tube recanalization can be safely performed laparoscopically after tube ligation with two-stitch technique. Laparoscopic reversal of the unilateral tube was performed to a 38-year-old woman who underwent unilateral salpingectomy due to ectopic pregnancy. At 3 days after surgery, tubal patency was determined via hydrotubation, and the results were satisfactory. The patient was assessed via hysterosalpingography after 2 months to confirm the patency of the anastomosed fallopian tube, and no tube obstruction was noted. The two-stitch procedure can reduce surgical time and prevent the possibility of new scar formation compared to traditional four-stitch approach.
\end{abstract}

https://drive.google.com/open?id=1yLWjT484__LE4jGfiC4wGYo-iRrRdM86

\section{Hosted file}

bjog.doc available at https://authorea.com/users/302700/articles/432796-laparoscopicreversal-of-tubal-sterilization-a-demonstration-video 

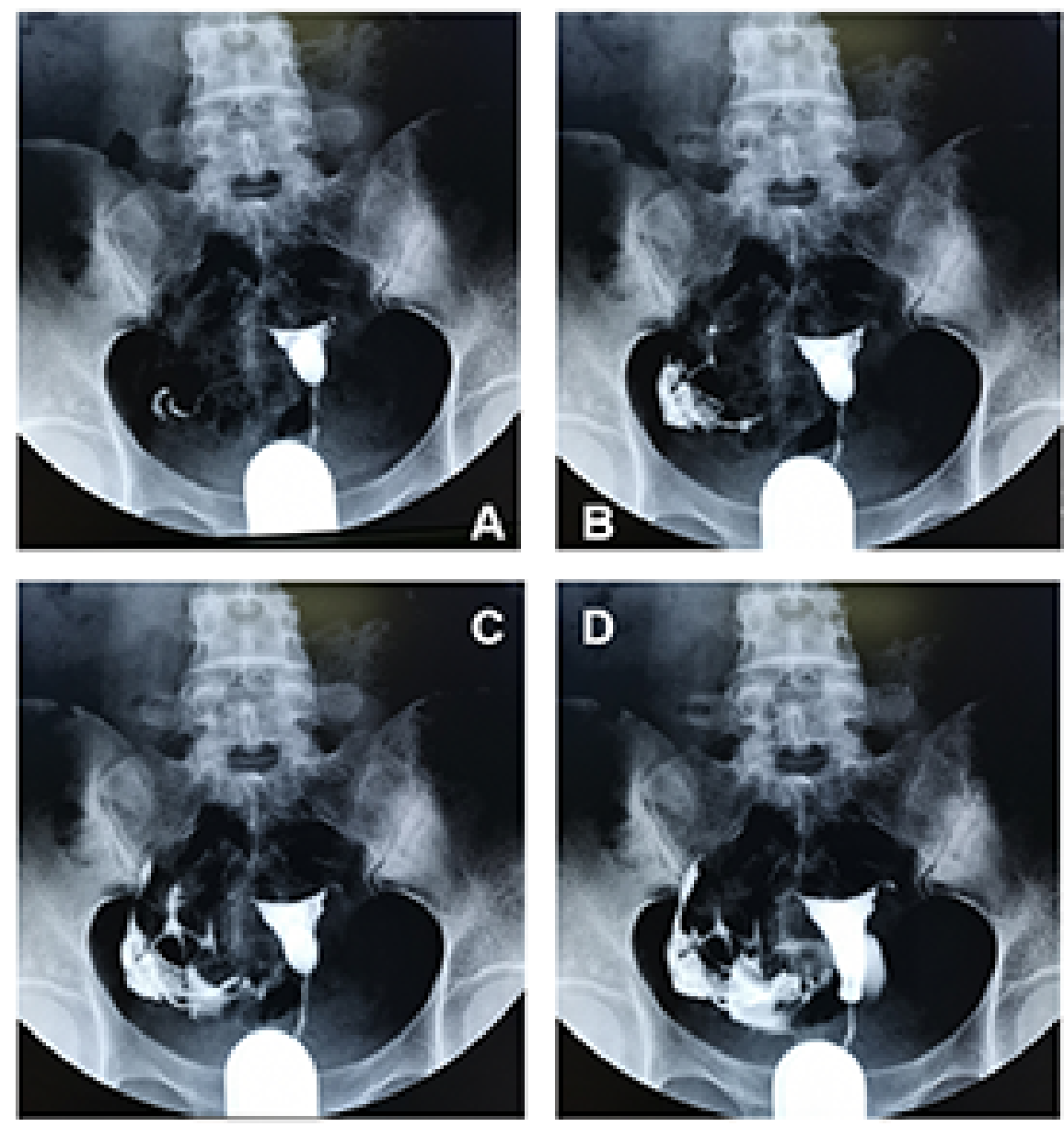